\title{
La complainte funèbre chez les troubadours et chez Saint Bernard
}

\author{
Brigitte Saouma \\ Centre d'Études et Recherches Occitanes - CEROC/Sorbonne
}

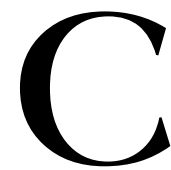

omparer la deploratio chez saint Bernard de Clairvaux avec les planhs des troubadours peut paraître risqué, sinon incongru, dans la mesure où Bernard pleure des moines et les troubadours des chevaliers, donc des laïcs. Or, dans la société médiévale, clercs et chevaliers formaient des groupes sociaux nettement différenciés. On s'est donc demandé si ces différences qui sont présentes dans les planhs, ne recouvraient pas des analogies. En d'autres termes, on a voulu savoir si, à travers l'éloge du défunt, le laïc et le clerc étaient si différents l'un de l'autre, qu'on avait coutume de l'affirmer ou si certaines de leurs qualités morales ne se rejoignaient pas. Une autre question se pose. Pourquoi avoir choisi saint Bernard, plutôt qu'un autre théologien? Parce que selon les termes du P. Leclercq:

Saint Bernard, en ce domaine comme tant d'autres ne représente pas que lui seul. Il est enraciné dans une tradition spirituelle et culturelle, celle du monachisme et il l'enrichit à son tour. ${ }^{1}$

Dans les deux groupes de textes, que représentent donc les planhs et les sermons de saint Bernard, l'éloge du défunt est présent ; il ne s'agit pas de l'éloge de ses actes, mais de ses qualités morales. La mention de cellesci, n'est pas suivie, en général, du récit d'actes précis les illustrant. Chez Bernard, cependant, on trouve une allusion à une belle action de Malachie. ${ }^{2}$

${ }^{1}$ J. Leclercq. "La joie de mourir selon saint Bernard de Clairvaux", in "Dies illa." Death in the Middle Ages, Proceedings of the 1983 Manchester Colloquium, cur. J. H. M. Taylor, Liverpool, 1984.

${ }^{2}$ In transitu sancti Malachiae Episcopi, 6, LL. 16-18, p. 421, in: Sancti Bernardi opera, Sermones II, Èd. J. Leclercq, H. Rochais, v. 5, Editiones Cistercienses. 
La générosité semble être la principale qualité du défunt, tant chez Bernard: Hilaris dator, petitor rarus, acceptorverecundus ${ }^{3}$ (Il donnait avec bonheur, demandait rarement et ne recevait qu'avec embarras) que chez les troubadours:

Quar el dava e metia, Que tot quan aver podia

El metia en boban, Quel mon bome non avia Quel sembles de donar tan. ${ }^{4}$

(Car il donnait et dépensait, / de sorte que tout ce qu'il pouvait avoir, / il le mettait dans le faste. / Et il n'y avait pas, au monde un homme / qui lui ressemblât pour donner autant).

La générosité telle qu'elle est décrite par les troubadours exclut que le seigneur reçoive quelque chose en retour de ses dons. En effet, possédant le pouvoir et l'argent, il prouve sa générosité en donnant abondamment sans rien réclamer en retour et même en refusant toute reconnaissance. Chez Bernard, le moine est généreux de manière analogue; il donne sans compter et ne demande rien en retour. La générosité consiste donc, tant pour les troubadours que pour Bernard, à penser à autrui avant de penser à soi. Le seigneur la pratique parce qu'il est riche et puissant, le moine la pratique par charité.

La seconde qualité reconnue au défunt est la douceur. Chez Bernard la douceur permet de supporter toutes les adversités sans jérémiades et sans rancune. Il nous dit à propos de Malachie:

Nam in spiritu lenitatis dura quaelibet et adversa aequo animo tolerabat ${ }^{5}$

(En effet, c'est grâce à l'esprit de douceur, qu'il peut supporter toutes les adversités et les choses les plus pénibles avec une âme égale).

${ }_{3}^{3}$ De sancto Malachia, 2, LL. 16-17, p. 51, in: Sancti Bernardi Opera, op. cit., Sermones III, Romae, 1970.

${ }^{4}$ Raimon Gaulcem de Bèziers, Quascus planh le sieu damnage, p. 11, in: Les troubadours de Bèziers, Èd. G. Azais, 2e Ėdition, Bèziers, 1869. Trad. modifiée.

${ }^{5}$ In transitu sancti Malachiae... op. cit., 7, LL. 5-6, p. 422. Cf. In: Obitu domni Humberti, 2, LL.13-15, p. 442, in Sancti Bernardi Opera, op. cit., vol.V, Sermones II, Romae, 1968. 
Le moine sait que celles-ci ne sont pas l'oeuvre de Dieu. Par conséquent, il ne saurait lui en attribuer la responsabilité. En revanche, c'est Dieu qui donne la force de les affronter. Pour Johan Estève de Béziers, le vicomte Amalrix est doux et loyal. ${ }^{6}$ La douceur du seigneur a-t-elle la même signification que celle du moine? Les troubadours, du moins dans les textes cités, ne donnent pas une véritable définition de la douceur du seigneur.

Cependant on rencontre chez Guiraut de Bornelh une allusion à la douceur telle que la définit Bernard :

\section{E qui-l parles iradamen, Saupra passar Ab fol de ma l'esmanza, Si bel. tires, alegramen, Si tot se tom' ir'e tenzars Saber en sen d'enfanza?}

(Si quelqu'un lui parlait avec colère, / il savait ne pas tenir compte / de la chose venant d'un fou mal éduqué, / quoique sous l'influence de la colère et de la discussion, / un être intelligent puisse avoir l'esprit d'un enfant.)

La troisième qualité du défunt, tant chez Bernard que chez les troubadours, est l'humilité: Erat ergo bumilis corde. ${ }^{8}$ (Il était humble de coeur) nous dit Bernard à propos d'Humbert et Rie d'orguelh, rie d'umilitat ${ }^{9}$ (Il était plein d'orgueil et plein d'humilité) nous dit Guillaume de Béziers à propos du vicomte de Béziers. L'humilité du moine consiste à savoir que tout le mal qu'il peut faire ou qu'il peut lui advenir vient de lui-même et non de Dieu. En revanche tout le bien qu'il peut faire ou qui peut lui advenir a son origine en Dieu. ${ }^{10}$ Retrouve-t-on la même notion chez les

\footnotetext{
${ }^{6}$ Johan Estève, Aissi quo.1 malanans, op. cit., V, v. 59, p. 116.

${ }^{7}$ Giraud de Borneil, N 77 Plaing e sospir V, v. 50-55, p.410, in: The Cansos and sirventes of the Troubadour Giraut de Borneil: a critical edition, Éd. R. V. Sharman, Cambridge, New York, 1989.

${ }^{8}$ In obitu... op. cit. 2, L. 13, p. 442.

${ }^{9}$ Guillaume de Bèziers, Quascus plor... op. cit., p. 125. Cf. Aimerie de Peguilhan, Era par ben... op. cit., 1, v. 6, p. 81.

${ }^{10}$ Sermones super Cantica Canticorum, 32, 5, LL 14-15 p. 229, in Sancti Bernardi opera, op. cit. vol. 1, Romae, 1957.
} 
troubadours? On ne peut l'affirmer avec certitude car ils ne la définissent pas dans les textes cités. On peut supposer, à la lecture de ces vers de Guiraut de Bornelh:

\section{Als franx parlava franchamen, Si tot s'er' orgoillos e cars \\ Lai ont hom se bobanza. ${ }^{11}$}

(Aux gens doux, il parlait avec douceur, / bien qu'il fût hautain et sévère pour les vantards)

que l'humilité du seigneur consistait à oublier son rang et tout ce qui en résultait, honneur, richesses et gloire pour se comporter avec tous comme les circonstances et le caractère de chacun l'exigeaient. Attitude que l'on retrouve chez Bernard dans la louange de Malachie:

\section{Et quidem infirmus infirmis, sed nibilominus potentibus potens, superbis resistebat, tyranos verberabat, regum magister ac principurn. ${ }^{12}$}

(On le voyait faible avec les faibles mais sans rien d'excessif. Il résistait aux orgueilleux / il fustigeait les tyrans, / et il donnait des leçons aux princes et aux rois).

Il existerait donc tant chez les troubadours que chez Bemard, une humilité qui serait fondée sur le respect universel de l'être, créature de Dieu quelque soit sa condition sociale. L'humilité du défunt le placerait donc au même rang que le plus démuni des hommes, sachant que s'il possède davantage c'est par la grâce de Dieu et de Dieu seul.

La quatrième qualité du défunt est celle de posséder le savoir. De quel savoir s'agit-il? Chez Bernard, le savoir serait plutôt la sagesse acquise par la prière quotidienne, au contact de Dieu. Sagesse qui permet d'éviter les excès et de rester dans une juste mesure en toutes choses:

Nam et zelans erat, nec deerat scientia, zeli ipsius moderatrix ${ }^{13}$

\footnotetext{
${ }^{11}$ Guiraud de Borneih, Plaing e sospir, op. cit. VI, vv. 64-66, p. 411.

${ }^{12}$ De sancto Malachia, op. cit. 2, LL 20-21 p. 51.

${ }^{13}$ Idem, L.19.
} 
(S'il avait un grand zèle, le zèle chez lui n'allait pas sans la science qui en est la modératrice).

Il semble qu'il en soit de même chez les troubadours qui louent à la fois le sen et la mezura ${ }^{14}$ du défunt.

La cinquième qualité du défunt est son ardeur au travail: Rarissime unquam sollemnibus vigiliis, quas tamen non raro anticipabat rarissime ceteris horis psallentium choro inventus est defuisse, nec nisi ea necessitate ut ex instanti responsum mortis habens, infirmitas sequeretur. ${ }^{15}$ (Il ne manqua que bien rarement, si tant est qu'il y ait jamais manqué, aux veilles solenelles, qu'il anticipait même quelquefois; rarement aussi, il se tint loin du choeur, quand les autres y étaient occupés), nous dit Bernard à propos de Malachie.

$$
\begin{aligned}
& \text { e sabia far son dever } \\
& \text { mielhs que lunbs que-n remanha say, }{ }^{16}
\end{aligned}
$$

(Et savait faire son devoir mieux qu'aucun qui soit demeuré ici).

nous dit Johan Estève de Béziers à propos de Guillaume de Lodève. Cet éloge est l'hommage à un défunt qui, par son travail contribuait au développement et à la pérennité de la société dans laquelle il vivait. Le défunt avait donc un rôle social reconnu. En effet, ni les troubadours, ni Bernard ne font l'éloge d'un défunt marginal, moine errant ou mendiant. Ce rôle social consistait, pour le moine à prier et pour le seigneur à combattre; et à protéger les siens. Les troubadours font du reste l'éloge des qualités guerrières du seigneur défunt:

\footnotetext{
${ }^{14}$ Aimeric de Belenoi, Ailas! per que viu lonjamen ni dura, IV, v. 26, p. 115, in Poésies du troubadour Aimeric de Belenoi, Éd. M. Dumitrescu, Paris, 1935. Bartolome Zorzi CLXXI) Sil monz fondes a maravilha gran, III, v. 24, p. 261, in Poesie provenzali storiche relative all'Italia, vol. 2, Éd. V. de Bartholornaeis, Roma, 1931.

${ }^{15}$ In obitu... op. cit., 3, 1. 12-15, p. 443.

${ }^{16}$ Johan Estève, Planhen ploran ab desplayer, II, vv. 11-12, p. 119, in Le poesie del trovatore Johan Estève, op. cit.
} 
Seingner, en vos non era res a faire, que totz lo mons vos avi'elegut Pel meillor rei que anc portes escut E-1 plus ardit e-1 meillor torneiaire Deus lo temps Rolan, ${ }^{17}$

(Seigneur, il n'y avait rien à reprendre en vous, car tout le monde vous avait choisi pour le meilleur roi qui eût jamais porté l'écu et le plus audacieux et le meilleur jouteur depuis le temps de Roland).

nous dit Bertrand de Born à propos de Henri le Jeune. Cependant, Bernard évoque aussi son idéal de la chevalerie, dans son éloge de la nouvelle milice:

\author{
Et in victu et in vestitu cavetur omne \\ superfluum, soli necessitati consulitur. \\ Vivitur in communi, plane iucunda et sobria \\ conversatione, absque uxoribus et absque \\ liberis. Et ne quid desit ex evangelica \\ perfectione, absque omni proprio habitant \\ unius moris in domo una, solliciti servare \\ unitatem spiritus in vinculo pacis. ${ }^{18}$
}

(Soit dans les habits, soit dans la nourriture, ils évitent toute superfluité et se bornent au strict nécessaire. Ils vivent rigoureusement en commun dans une douce mais modeste et frugale société, sans épouses et sans enfants ; bien plus suivant les conseils de la perfection évangélique, ils habitent sous un même toit, ne possèdent rien en propre et ne sont préoccupés que de conserver entre eux l'union et la paix).

Cet idéal de chevalerie est très proche de l'idéal monastique que Bernard valorise. Cependant Bernard reste conscient de la différence entre les deux rôles lorsqu'il écrit à propos des qualités guerrières du chevalier:

\footnotetext{
${ }^{17}$ Bertran de Born, N'l 3, Mon chan fenis ab dol et ab maltraire, op. cit. IV, vv 43-47, p. 242, in L'amour et la guerre. L'oeuvre de Bertran de Born, op. cit.

${ }^{18}$ Liber ad milites Templi de laude novae militiae, cap. 4, 1. 4-8, p. 220, in Sancti Bernardi Opera, op. cit., v. III, Romae, 1963.
} 
Denique, quod ipsi saepius cetiusque

experimini, tria esse praecipue necessaria

praelianti, ut scilicet stenuus

industriusque miles et circonspectus sit

ad se servandum, et expeditus ad

discurrendum, et promptus ad

feriendum ...19

(Mais nous savons, par une expérience fréquente et certaine que le soldat qui marche au combat n'a besoin que de trois choses, d'être vif, exercé et habile à parer les coups, alerte à la poursuite et prompt à frapper).

La chevalerie idéale pour Bernard a donc toutes les caractéristiques du soldat et toutes les qualités du moine, du moins, en apparence. Car, malgré tout, Bernard, ne reconnait pas à la chevalerie du Temple les véritables prérogatives des moines. Il s'agit, en effet, de soldats désireux d'expier leurs fautes passées et non de religieux ayant entendu l'appel divin.

En conséquence, on peut affirmer que l'éloge du défunt chez Bernard et chez les troubadours se rejoint sur un certain nombre de points. Dans les deux cas, le défunt est généreux, humble, doux et bon travailleur. Bien entendu, les troubadours ne font pas l'éloge du moine et Bernard ne fait pas l'éloge du chevalier. Cependant, paradoxalement, Bernard détaille davantage les qualités guerrières du chevalier que ne le font les troubadours.

Parallèlement à la louange du seigneur défunt, les troubadours ont parfois pleuré leur dame défunte. C'est un hommage à une dame aimée que la mort a arrachée au troubadour:

q'anc no fo hom ne vis tan avinen.

Qi aura mais tan bel captenemen?

qe val beutatz, ni bons prez mentagus,

ni qe val sens, honors ni solaz gais

genz acuillirs ni nuills cortes essais, ni qe valon franc dich ni faich prezan?20

${ }^{19}$ Idem, cap. 11, p. 216, 1113-16.

${ }^{20}$ Pons de Capdueil, De totz chaitus sui en aicel qe plus, p. 121, vv. 20-25, in "Pons de Capdueil and Azalais de Mercuor: a study of the planh", Nottingham, Medieval Studies, 2, 1958, p. 119-131. 
(Car jamais homme, ne vit plus avenante. Qui aura désormais une si belle manière d'agir? Que valent beauté ou valeurs reconnues, raison, joyeuses conversations, nobles accueils, et essais courtois?).

Cet hommage est différent de celui du seigneur dans la mesure où il ne met pas en valeur les mêmes qualités. En effet, la dame est courtoise sait accueillir, converser gaiement, et possède de belles manières. Cela n'a pas grand-chose à voir avec les qualités morales du seigneur défunt. En effet, d'aprés ces vers, la dame ne posséderait que des qualités sociales. Or, ce n'est pas le cas dans les chansons d'amour. Ce qui n'empêche pas le poète de clamer que la mort est cruelle, car pour Bernard, comme pour les troubadours, la mort est cruelle. Elle ôte au troubadour, comme au moine, le bénéfice de la vie auprès d'un être cher:

perdut avem en vos la fon

d'on tug veniam jauzion ${ }^{21}$

(Nous avons perdu en vous la fontaine de laquelle nous revenions joyeux)

dira Guilhem Augier Novella à propos de Raimon Roger Trencavel.

Qui a nobis susceptus est tamquam verus ab

Occidente veniens, visitans nos Oriens ex alto $^{22}$

(Quand il est arrivé chez nous, du fond de l'occident, nous l'avons reçu comme le vrai soleil levant, venu du haut du ciel pour nous visiter).

dira Bemard à propos de Malachie. Plus le disparu a été précieux, plus sa mort est ressentie comme injuste. Cette mort cruelle est un adversaire d'autant plus redoutable que nul ne peut la combattre. Tout un chacun est totalement impuissant devant elle. Mais si les troubadours se contentent de vitupérer contre elle, ${ }^{23}$ Bernard lui rappelle que la fin des

\footnotetext{
${ }^{21}$ Guilhera Augier Novella, Quascus plor e planh, op. cit., V, vv. 49-50, p. 92. ${ }^{22}$ Vita sancti Malachiae, cap. XXXI, 70, LL 6-7, p. 374, in Sancti Bernardi opera, op. cit., vol. 111.

${ }^{23}$ Johan Estève Aissi quo.1 malanans, op. cit. Ill, vv. 25-30, p. 115.
} 
temps verra sa disparition. ${ }^{24}$ L'action de la mort est funeste car elle prend l'être cher et frappe aveuglément en s'emparant du meilleur. ${ }^{25}$

Cependant, la mort n'exerce son action que sur le corps, tant chez les troubadours que chez Bemard. Les troubadours ne parlent pas d'une mort de l'âme ; au contraire, ils insèrent une prière pour l'âme du disparu dans leurs planhs. Par conséquent la victoire de la mort est partielle puisqu'elle ne s'effectue que sur le corps. Que l'âme reste vivante est une grande consolation, surtout chez Bemard. En effet, la mort du moine représente la porte de la vie béatifique; elle permettra l'Union avec $\operatorname{Dieu}^{26}$ qui est le but ultime de la vie spirituelle. La mort du moine est donc considérée comme une délivrance. C'est la mort joyeuse, la mort précieuse.

\section{Non est igitur quod causeris tu de nostra quasi subtracta tibi praesentia, cui affatim sui suorumque copiam Dominus maiestatis indulsit ${ }^{27}$}

(Tu n'as point à te plaindre de nous avoir quittés puisque le seigneur t'accorde à volonté sa propre présence et celle de ses amis).

La mort du moine est donc "attendue sans peur" elle est accompagnée de sérénité et même de beauté. ${ }^{28}$ Cette mort est pleine de sécurité car elle a été préparée tout au long de la vie. En effet, le moine s'est consacré à Dieu, gravissant un à un les degrés du cheminement spirituel qui mène à la béatitude. Dans une lettre adressée à un jeune homme hésitant à entrer dans les ordres, Bernard écrit:

${ }^{24}$ In obitu ... op. cit., 1, L. 15, p. 441.

${ }^{25}$ Bertran de Born, NI 13, Mon chan fenis... op. cit. IV, v 45, p. 242; Guilhern Augier Novella, Quascus plor... op. cit. V, v 47-48, p. 92; Guillaume de Béziers, Quascus plor e planh... op. cit., v 54, p. 125; Johan Estève, Aissi quo.1 malanans, op. cit. 111, vv 31-32, p. 115; Raimon Gaulcem de Béziers, Abs grans trebalhs, op. cit. p. 35, Quascus planh... op. cit., p. 11.

${ }^{26}$ Sermones super Cantica Canticorum, op. cit., t.1 sermo 2, 2, LL17-20, p. 9.

${ }^{27}$ Idem, sermo 26, 5, LL5-7, p. 173.

${ }^{28}$ J. Leclercq, "La joie de mourir"... op. cit., p. 195. 
Volo te mortem, etsi non effugere, certe vel non timere. ${ }^{29}$

(Puisque tu ne peux éviter la mort, je veux que du moins tu ne la craignes pas).

Par conséquent, qui doit redouter la mort sinon le pécheur? Et le pécheur est essentiellement un seigneur, un de ces seigneurs qui combattent pour une vaine gloire ${ }^{30}$ et qui sont des voleurs, des parjures, des scélérats et des maris infidèles. Bernard ne trouve pas assez de mots pour vitupérer contre ces seigneurs et il ne manque pas de leur rappeler que toutes leurs batailles n'auront servi qu'à les mener à la véritable mort, la mort de l'âme.

Quis ergo, o milites, bic tain stupendus error, quis furor bic tain non ferendus, tantis sumptibus ac laboribus militare, stipendis vero nullis, nisi aut mortis, aut criminis? ${ }^{31}$

(Combien étrange, n'est donc point votre erreur, ou plutôt quelle n'est pas votre insupportable fureur, ô soldats de faire la guerre avec tant de peine et de frais pour n'en être payés que par la mort ou par le péché ?).

Bien entendu Bernard n'exprime aucun regret pour la disparition de l'un de ces soldats; il n'éprouve aucune estime et n'a aucune considération pour eux. Mais c'est à cette chevalerie que les troubadours adressent leurs planhs. C'est pour elle qu'ils implorent la clémence divine. Cela d'autant plus qu'ils chantent la guerre séculière la guerre que Bernard condamne avec tant de fougue. Il est d'autant plus surprenant, par ailleurs, de voir le troubadour Bertran de Bom chanter la guerre avec passion $^{32}$ et parallèlement pleurer un seigneur mort à la guerre comme si la mort n'était pas la conséquence logique de la guerre. Cependant,

\footnotetext{
${ }^{29}$ Epistola CV, L. 16, P. 264, in Sancti Bernardi opera, op. cit., v. Vil, Romae, 1974. ${ }^{30}$ De laude... op. cit., cap. 11, 3, L.22, p. 216.

${ }^{31}$ Epistola CCCLXIII, 4, L.5, p. 314, in Sancti Bernardi opera, op. cit., v. VIII, Romae, 1977. Cf. De laude... op. cit. cap. V, 10, LL.5-6, p. 223.

${ }^{32}$ De Laude... op. cit., cap. II, 3,1. LL5-7, p. 216.
} 
Bernard qui se fait le porte-parole de Dieu, présente à cette chevalerie le moyen de se racheter et par conséquent de mourir avec sécurité. Ce moyen passe par la guerre, certes mais une guerre qui est aussi source de rédemption pour ceux qui ne connaissent que le métier des armes. Ce moyen consiste à s'engager dans la chevalerie du Temple, avec les soldats du Christ. En effet, les chevaliers du Temple combattent en sécurité car ils se sont mis au service du Christ. Le bénéfice de leur guerre en revient donc au Christ et non à eux mêmes. La guerre qu'ils font est donc une guerre juste:

Non enim sine causa gladium portat : Dei

enim minister est ad vindictam

malefactorum, laudem vero bonorum. ${ }^{33}$

(Ce n'est pas en vain qu'il porte l'épée à; il est le ministre de Dieu, en punissant ceux qui font de mauvaises actions et en récompensant ceux qui en font de bonnes).

Ce chevalier tue donc avec sécurité les infidèles et meurt avec encore plus de sécurité:

At vero Christi milites securi praeliantur praelia Domini suinequaquam metuentes aut de bostium caede peccatum, aut de sua nece periculum, quandoquidem mors pro Christo vel ferenda, vel inferenda, et nibil habeat criminis, et plurimum gloriae mereatur. ${ }^{34}$

(Les soldats du Christ combattent en pleine sécurité les combats de leur seigneur, car ils n'ont point à craindre d'offenser Dieu en tuant un ennemi et ils ne courent aucun danger s'ils sont tués eux-mêmes, puisque c'est pour JésusChrist qu'ils donnent ou reçoivent le coup de la mort).

Bernard engage même ces chevaliers à mourir tués par les infidèles, plutôt qu'à tuer ces mêmes infidèles car ainsi ils sont sûrs d'accéder à la vie éternelle. ${ }^{35}$ La sécurité des Templiers rejoint la sécurité du moine dans

${ }^{33}$ De laude, op. cit., cap. III, 4, 1. 9-10, p. 217.

${ }^{34}$ Idem, 1. 1-5.

${ }^{35}$ Idem. 
la mort. Néanmoins, Bernard ne leur accorde pas la mort joyeuse car, manifestement, ils n'en sont pas dignes malgré leur mort au service du Christ. En effet leur vie aura été consacrée à la guerre et non à la prière. Mais les troubadours, eux, ont rendu hommage à un croisé, le roi Saint Louis:
Abs grans trebalhs et ab grans marrimens
Veyrem hueymais cristiantat estar,
Pus mortz es selh qu'era del mon ses par,
Que valia sobre totz los valens,
Qu'era de cor per Jhesu-Crist issitz
Del sÓeu pays contrals fals Turcx aunitz, ${ }^{36}$

(Avec grandes peines et grandes douleurs, nous verrons désormais être la chrétienté puisque mort est celui qui était au monde sans égal, qui était vaillant au-dessus de tous les vaillants, qui était courageusement pour Jésus-Christ sorti de son pays contre les perfides Turcs honnis).

Raimon Gaulcem de Béziers évoque la peine et la difficulté qu'ont pu éprouver ces chevaliers à partir pour une guerre dont ils ne tireraient pas profit. Un point de vue qui est contraire à celui de Bemard et qui marque la différence entre seigneurs et clercs lorsqu'ils parlent de la guerre. Pourtant, Bemard a utilisé le goût du profit des chevaliers pour les inciter à entrer dans la chevalerie du Temple. En effet, ils y gagneraient le salut et la vie éternelle.

Tant Bernard que les troubadours ont évoqué la mort du Christ pour le rachat des péchés.

\begin{abstract}
Cum ergo bac gemina morte secundum manique naturam bomo damnatus fuisset, altera quidem spirituali et voluntaria, altera corporali et necessaria, utrique Deus homo una sua corporali ac voluntaria begnine et potenter occurrit, illaque una sua nostrain manique damnavit ${ }^{37}$
\end{abstract}

\footnotetext{
${ }^{36}$ Raimon Gaulcem de Béziers, Abs grans trebalhs... op. cit. p. 34-35 in Les troubadours de Béziers, op. cit., - Cf. Daspols, fortz tristors es e salvaj' a retraire, vv 3-4, p. 41, in Les derniers troubadours de la Provence, Éd. P. Meyer, Paris, 1871. ${ }^{37}$ De laude... op. cit., cap, XI, 20, LL.3-6, p. 231.
} 
(L'homme donc se trouvant condamné à une double mort dans sa double nature, l'une spirituelle et volontaire, l'autre corporelle et forcée, homme - Dieu a remédié à l'une et à l'autre avec autant de bonté que d'efficacité par sa mort corporelle et volontaire, et en mourant une fois, il a tué nos deux morts).

Bel seigner Dieus, cui non platz

mortz de negun pechador,

anz per aucire la lor

sofritz vos la vostr'en patz, ${ }^{38}$

(Beau sire Dieu, puisque vous ne voulez la mort d'aucun pécheur et que, au contraire, pour détruire la leur, vous souffrîtes la vôtre en paix).

Certains troubadours comme Guilhem Augier Novella ont même assimilé la mort de leur seigneur à la mort du Christ:

... e pus Dieus mort pres

per nos a salvar, semblans es

de lui, qu'es passatz al sieu pon

per los sieus estorser l'aon. ${ }^{39}$

(Puisque Dieu est mort pour nous, qu'il vienne en aide à celui qui lui ressemble, celui qui est passé par le même pont pour sauver les siens).

En effet, le péché a été introduit dans l'humanité dès l'origine. Mais le Christ qui en était dépourvu, a souffert la mort humaine pour en sauver les hommes. Il en a ainsi délivré l'humanité. Il a pu réaliser cela parce que sa mort a été volontaire et spontanée. C'est un acte de liberté totale. Il est mort par amour, par pitié, par miséricorde. Il a été capable de mourir parce qu'il était homme et de se libérer de la mort parce qu'il était Dieu. ${ }^{40}$ Presque tous les troubadours ont inséré dans leurs planhs une prière pour le défunt. Cette prière est destinée à implorer Dieu d'accueillir au

\footnotetext{
${ }^{38}$ Folquet de Marseille, XVII. Si cum cel qes tan greujatz, op. cit., vv. 56-59, p. 76. Trad. modifiée.

${ }^{39}$ Guilhem Augier Novella, Quascus plore planh... op.cit., Il, vv. 17-20, p. 90.

${ }^{40}$ De laude... op. cit., cap XI, 25, LL. 15-16, p. 234.
} 
paradis le seigneur disparu. Or le mort est paré de toutes les vertus, ce qui devrait lui assurer la miséricorde divine. Il est donc probable que les troubadours devaient sentir que, même si le défunt avait fait pénitence et regretté avec humilité toutes ses fautes, Dieu laissait quand même les vivants dans une incertitude partielle à son sujet. Cette incertitude devait être d'autant plus réelle qu'ils n'ignoraient sans doute pas que l'Église condamnait la guerre séculière, la principale source de mortalité parmi les chevaliers. En effet, les troubadours connaissaient le problème du salut et de la vie éternelle puisqu'ils l'évoquent dans leurs chansons de croisade.

Dans ces deux séries de textes, on constate donc que la mort est l'occasion de valoriser le modèle social du disparu en écartant avec soin tout ce qui pourrait en ternir l'image. Le défunt est donc un héros, un modèle à imiter tant chez les troubadours que chez Bernard. Il n'est donc plus un individu mais l'exemple auquel on se reportera. En effet, à travers lui ce sont les qualités qui cimentent le groupe social qui font l'objet de l'hommage car elles ne doivent en aucun cas disparaitre avec le mort. Elles doivent au contraire lui survivre, magnifiées, à l'intérieur du groupe social. Un chevalier doit être preux, valeureux au combat, car il assure la défense de son territoire et des personnes qui dépendent de lui.

En ce sens, sa mort est une perte. Mais on invite le successeur, implicitement, à faire preuve des mêmes qualités. Le moine qui meurt est une perte pour la communauté, mais sa mort n'en demeure pas moins un exemple tout comme sa vie l'a été ou aurait dû l'être. L'antagonisme entre les deux groupes sociaux se fonde essentiellement sur la guerre. En effet, les troubadours la chantent quand les moines ne l'acceptent pas. Mais, il importe de remarquer que Bemard ignore superbement le rôle de défense et de protection qu'ont joué les seigneurs dans la société féodale; rôle dont les moines ont largement profité puisqu'il leur a permis de vivre à l'abri des monastères. En effet, il est fort probable que privés de la protection des seigneurs, ils auraient été contraints de prendre les armes pour défendre leurs possessions.

En revanche, on a pu remarquer que les qualités du défunt sont analogues, tant chez Bernard que chez les troubadours, hormis les qualités d'homme de guerre, bien sûr. Peut-on en conclure que la séparation entre clercs et chevaliers n'était qu'apparente? 


\section{Résumé}

L'éloge des qualités morales du défunt est présent tant dans les plagnes des troubadours que dans les sermons de saint Bernard. La principale qualité commune aux moines et aux seigneurs est la générosité viennent en suite la douceur, l'humilité et la sagesse. Le défunt pleuré par les troubadours est généralement un seigneur bien plus rarement une dame, on exalte donc ses qualités guerrières, en revanche pour Bernard les chevaliers ne sont dignes de louange que lorsqu'ils se mettent au service du Christ. Dans les deux groupes de textes la mort est cruelle parce qu'elle frappe le meilleur des hommes. Mais elle reste la mort du corps et non celle de l'âme. Le défunt devient un héros, un modèle, l'exemple auquel on se reportera.

\section{Resumo}

O elogium das qualidades do defunto está presente, tanto nos prantos dos trovadores, como nos sermões de São Bernardo. A principal qualidade comum aos monges e aos senhores [feudais] é a generosidade; em seguida vêm a doçura, a humildade e a sabedoria. O defunto pranteado pelos trovadores é geralmente um senhor e, muito raramente, uma dama; exaltam-se, assim, as suas qualidades guerreiras; para Bernardo, no entanto, os cavalheiros não são dignos de louvação senão quando se colocam a serviço de Cristo. Nos dois grupos de textos a morte é cruel porque atinge o melhor dos homens. Ela é, porém, a morte do corpo e não a da alma. O defunto se torna um herói, um modelo, um exemplo ao qual se deve imitar. 